\title{
SIRT6, oxidative stress, and aging
}

Cell Research (2016) 26:143-144. doi:10.1038/cr.2016.8; published online 19 January 2016

The role of oxidative stress in the aging process has been highly debated for decades and remains equivocal. $A$ new study published in Cell Research reports a novel role for the agingassociated SIRT6 deacetylase in the control of oxidative homeostasis in human mesenchymal stem cells.

Aging is a multifactorial process likely modulated by diverse molecular and cellular events, such as genome instability, epigenetic and transcriptional changes, molecular damage, cell death and senescence, inflammation, and metabolic dysfunction [1]. These processes are interconnected and understanding how each contributes to individual aspects of aging pathology remains largely unknown, making it challenging to tease out the triggering events during aging.

An enduring possible explanation for aging is the oxidative stress theory [2]. Although there is solid evidence showing that oxidative stress is a contributor to some aging pathologies, lifespan extension has yet to be consistently achieved through strategies to mitigate levels of oxidative damage [3]. It also remains unclear which tissues and cell types might be the most sensitive to oxidative stress during aging.

SIRT6 is one of the few genes known to regulate both longevity and progeria (a genetic disorder resembling accelerated aging) $[4,5]$. Specifically, ubiquitous SIRT6 overexpression leads to enhanced longevity, although this effect is seen only in males [6]. Conversely, mice lacking SIRT6 exhibit progeroid phenotypes [7]. Functionally, SIRT6 plays an important role in DNA repair, telomerase function, genomic stability, and cellular senescence [8]. Until now, however, SIRT6 has not been associated with oxidative stress. A new study from Pan et al. [9] links the deacetylase activity of SIRT6 to redox homeostasis in human mesenchymal stem cells (hMSCs) and suggests that this activity may regulate the longevity and progeria phenotypes (Figure 1).

Pan et al. [9] generated SIRT6deficient human embryonic stem cells (hESCs), differentiated them into hMSCs (SIRT6 ${ }^{-1-}$ hMSCs) and characterized their properties. Interestingly, these cells showed premature and progressively accelerated cellular senescence as demonstrated by several senescent markers and also exhibited elevation of reactive oxygen species (ROS), dysregulated redox metabolism, and increased sensitivity to oxidative stress. Contrary to a prior work performed in mouse embryonic fibroblasts (MEFs) [7], SIRT6-/- hMSCs did not exhibit enhanced genomic instability [9], suggesting that cell type differences may underlie the divergent phenotypes. Indeed, Pan et al. [9] found that transcriptional changes upon SIRT6 depletion in hMSCs were not recapitulated in human vascular endothelial cells.

Motivated to uncover the molecular mechanisms underlying the phenotypes of SIRT6 ${ }^{-/-}$hMSCs, Pan et al. [9] applied both genome-wide RNA sequencing and chromatin immunoprecipitation-sequencing analyses. The primary finding from these approaches is that SIRT6 acts as a transactivator for the cytoprotective transcription factor, nuclear factor erythroid 2-related factor 2 (NRF2), a critical redox sensor and regulator of antioxidant responses.
SIRT6 localizes to NRF2-associated promoters through direct association, where it directly recruites RNA polymerase II and indirectly creates a supportive environment for recruitment of RNA polymerase II by deacetylating H3K56 locally. Furthermore, the authors observed that overexpression of heme oxygenase (HO-1), an antioxidant gene downstream of NRF2, restored resistance to oxidative damage in SIRT6 ${ }^{-1}$ hMSCs, and improved the ability of these cells to engraft and proliferate in vivo. HO-1 downregulation also ac-

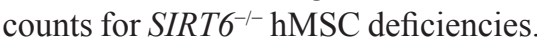
Thus, these data suggest that SIRT6 functions to positively modulate the NRF2-regulated antioxidant pathway in hMSCs, uncovering a novel mechanism for regulation of oxidative stress and possibly explaining links between the deacetylase activity and aging.

SIRT6 is a highly specific histone type 3 deacetylase that targets $\mathrm{H} 3 \mathrm{~K} 9$ and H3K56 and plays an important role in DNA repair, telomerase function, genomic stability, and cellular senescence [8]. Given that sirtuins such as SIRT6 are conserved $\mathrm{NAD}^{+}$-dependent deacylases, their function is intrinsically linked to cellular metabolism. In addition to being a $\mathrm{NAD}^{+}$consumer, SIRT6 modulates glucose metabolism by maintaining deacetylation of $\mathrm{H} 3 \mathrm{~K} 9$ in promoters of glycolytic genes and functioning as a co-repressor of HIF $1 \alpha$ [10]. Interestingly, SIRT6 ${ }^{-1-} \mathrm{hMSCs}$ exhibited elevation of H3K56Ac level as expected, but not H3K9Ac [9]. Again observed differences may reflect cell-type specificity, although species differences remain a possibility and further studies are needed to provide 


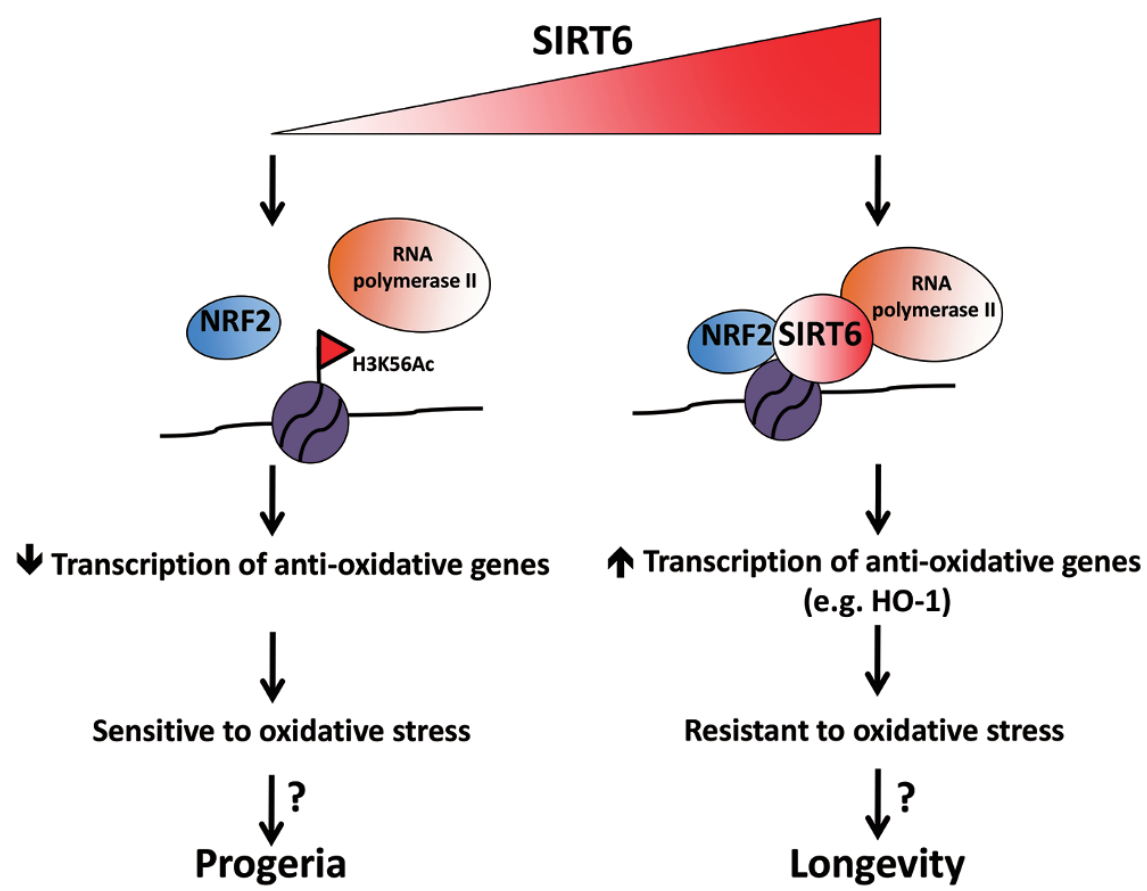

Figure 1 SIRT6 regulates longevity and progeria. The density of the red color indicates the concentration of SIRT6 protein. The arrows in this model indicate relationships that are yet to be firmly established in other cell types and variable mouse models.

clarification.

The relationship between aging, SIRT6-mediated deacetylation and redox biology is intriguing. In line with previous studies, SIRT6 may play a pivotal role in both longevity and progeria, and it would be interesting to study whether modulation of ROS production might underlie this response. It will be of interest to examine the SIRT6-NRF2HO-1 axis in vivo in the context of both SIRT6 overexpression-mediated lifes- lamins are required for SIRT6 activation and support SIRT6-mediated DNA repair [12]. As with many important studies, the findings of Pan et al. [9] raise important questions whose answers may functionally identify key components in aging regulation and accelerate progress towards a systemic understanding of human aging process.

\section{Chen-Yu Liao ${ }^{1}$, Brian K Kennedy ${ }^{1}$

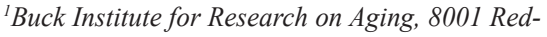 wood Blvd., Novato, CA 94945, USA \\ Correspondence: Brian Kennedy \\ Tel: +1-415-209-2040 \\ E-mail: bkennedy@buckinstitute.org}

\section{References}

1 Kennedy BK, Berger SL, Brunet A, et al. Cell 2014; 159:709-713.

2 Harman D. J Gerontol 1956; 11:298-300.

3 Perez VI, Bokov A, Van Remmen $\mathrm{H}$, et al. Biochim Biophys Acta 2009; 1790:10051014.

4 Liao CY, Kennedy BK. Cell Res 2012; 22:1215-1217.

5 Liao CY, Kennedy BK. Curr Top Dev Biol 2014; 109:249-285.

6 Kanfi Y, Naiman S, Amir G, et al. Nature 2012; 483:218-221.

7 Mostoslavsky R, Chua KF, Lombard DB, et al. Cell 2006; 124:315-329.

8 Tennen RI, Chua KF. Trends Biochem Sci 2011; 36:39-46.

9 Pan H, Guan D, Liu X, et al. Cell Res 2016; 26:190-205.

10 Zhong L, D'Urso A, Toiber D, et al. Cell 2010; 140:280-293.

11 Liu B, Ghosh S, Yang X, et al. Cell Metab 2012; 16:738-750.

12 Ghosh S, Liu B, Wang Y, et al. Cell Rep 2015; 13:1396-1406. 